\title{
Analysis of the Pierre Auger Fluorescence Data
}

\author{
Heiko Geenen ${ }^{1}$ for the Pierre Auger Collaboration ${ }^{2}$ \\ ${ }^{1}$ University of Wuppertal, Department of Physics, Gaußstr. 20, D-42097 Wuppertal, Germany \\ ${ }^{2} \mathrm{http}: / /$ www.auger.org/auger-authors.pdf
}

\begin{abstract}
The Pierre Auger Observatory is the largest extensive air-shower (EAS) experiment in the world and is still under construction. The final configuration will have detectors at two sites: in the northern (USA) and southern (Argentina) hemispheres. The aim of the experiment is to determine the energy, composition and origin of the UHE cosmic-rays above $10^{18} \mathrm{eV}$ using two complementary detection techniques: water Cherenkov detectors and fluorescence telescopes. The observatory at the southern site already contains more than 1000 (Sep. 2006) water Cherenkov detectors and 18 fluorescence telescopes in 3 telescope buildings. Completion of construction is planned for the end of 2007 with a final configuration of 1600 tanks and 24 telescopes.

In clear nights, this configuration permits the measurement of the lateral as well as the longitudinal development of an EAS (hybrid event). The fluorescence detector (FD) plays a major role not only in the calibration of the energy scale of the experiment, but also in the understanding of the systematics of the experiment by using the data itself.

This presentation focuses on the fluorescence telescopes of the Auger detector, on the performance and systematics of the FD reconstruction and event topologies possible with Auger FD.
\end{abstract}

\section{The CurRent Status of The Pierre Auger OBSERVATORY}

The southern site of the Pierre Auger Observatory is located in Malargüe, Province Mendoza, Argentina. In the Argentinian Pampa on an area of $3600 \mathrm{~km}^{2}, 1600$ water Cherenkov tanks are being installed with a spacing of $1.5 \mathrm{~km}$. By September 2006 more than 1000 tanks have been deployed. Fig. 1 shows the location of the southern site and the deployed area by September 2006. The water tanks form the so called surface detector (SD) array of Auger, that is surrounded by four fluorescence telescope sites: Los Leones, Los Morados, Loma Amarilla and Coihueco. The telescope buildings are divided in six bays. Each bay houses a Schmidt optic, a mirror and a $20 \times 22$ PMT pixel camera to monitor a field of view of $30^{\circ} \times 30^{\circ}$. The camera is read out by FADCelectronics and provides a time resolution of 100ns. The primary role of the fluorescence detectors (FD) is to measure the longitudinal development profile of air showers in the atmosphere by detecting the fluorescence light excited by the electromagnetic cascade of the EAS. This allows operation only in clear nights (10\% duty cycle). The longitudinal profile gives a direct calorimetric measure of the electromagnetic shower energy. Complementary the SD array can measure the lateral profile of the shower footprint. The set of showers that are measured by the combination of SD and FD (hybrid showers) can be used to calibrate the energy scale of the SD array in a model-independent way. Moreover, the FD data

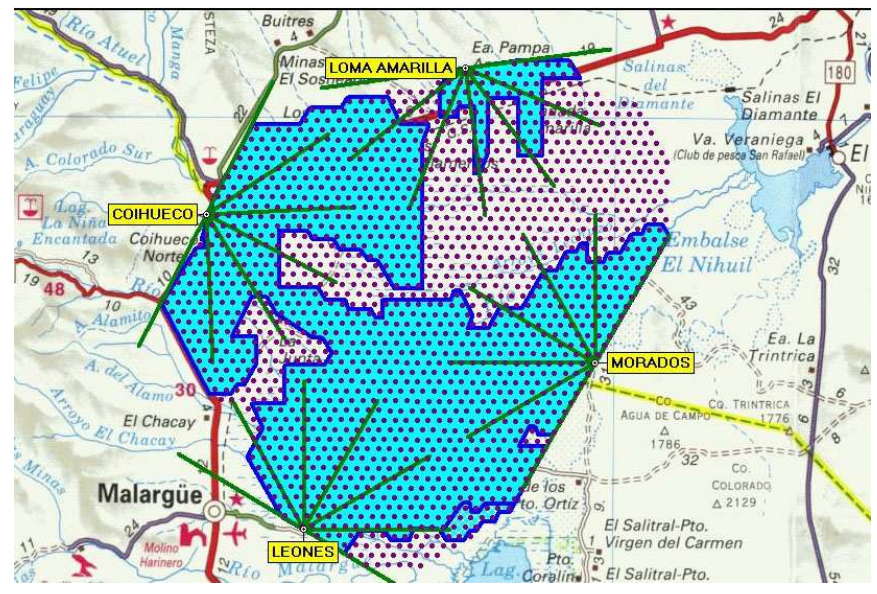

Fig. 1. The Pierre Auger southern site: $3600 \mathrm{~km}^{2}$ of Argentinian Pampa will be instrumented by 1600 water Cherenkov tanks of $1.5 \mathrm{~km}$ spacing. Each dot represents the position of a tank. The blue area shows the amount of tanks that have been deployed by September 2006. The surface detector area is surrounded by four fluorescence telescope sites: Los Leones, Los Morados, Loma Amarilla, Coihueco

can be used to understand and validate the systematics of the experiment. By September 2006 the telescope sites Los Leones, Los Morados and Coihueco were fully operational (Fig 2). The 4th site, Loma Amarilla, is under construction and expected to see first light early 2007. In addition the atmosphere above the experiment is continously monitored by many devices to guarantee good reconstruction quality.
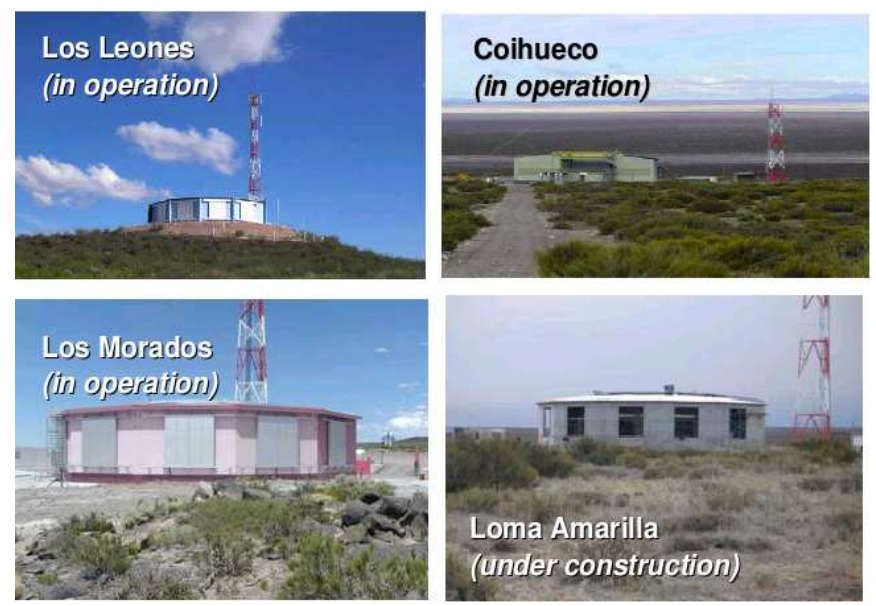

Fig. 2. The four fluorescence telescope sites: Los Leones, Los Morados, Loma Amarilla, Coihueco 


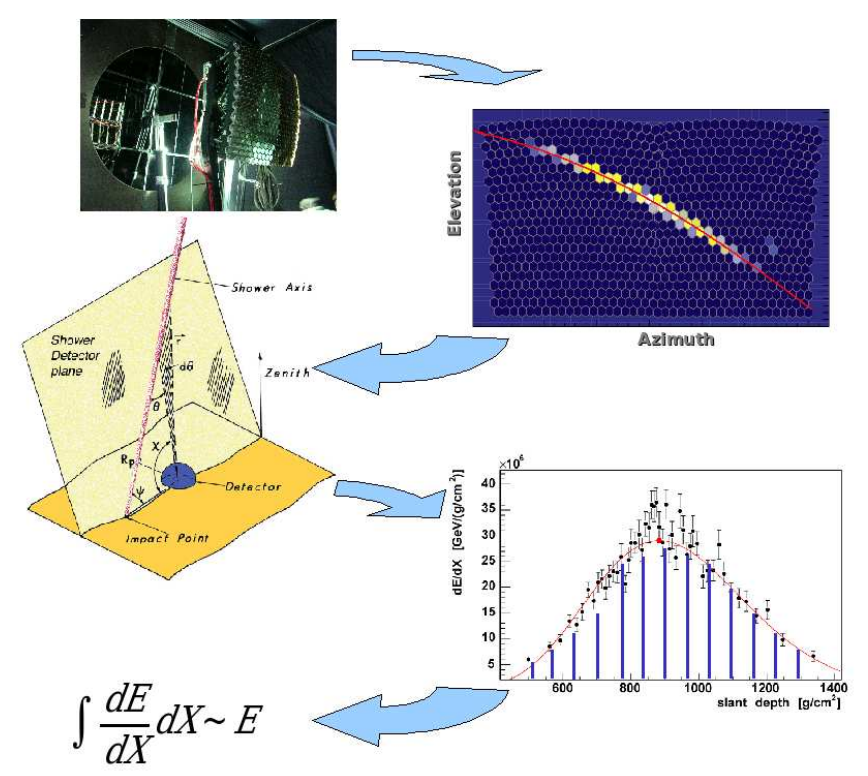

Fig. 3. Schema of the reconstruction method using FD-only information.

A LIDAR next to each telescope site continously measures the relevant Rayleigh scattering and absorption length in the field of view of the telescopes. Horizontal attenuation monitors shoot from one telescope to the other to measure the aerosol content near ground. A central Laser Facility shoots regularly up-going calibrated Laser shots. The signal is splitted and a part of the beam is injected via optical fiber to a tank. This does not only allow a calibration of the FD, but also a crosscheck of the timing between FD and SD. Star- and cloud-monitors measure the cloud coverage and atmospheric absorption to validate the data quality. Regular radio soundings provide the air density profiles.

\section{RECONSTRUCTION AND SYSTEMATICS}

A reconstruction schema using the FD information alone is shown in Fig. (3): The fluorescence light from the EAS has the clear signature of a down-going trace in the camera. A standard fitting procedure for one telescope starts with the determination of the plane containing the shower axis, that is defined by the camera trace, and the telescope itself. This shower detector plane (SDP) uses the PMT pointing directions weighted by the signal integral. Once the SDP is determined the exact timing informations of the signal arrival times can be used to fit the exact orientation of the shower axis in that plane. For a given geometry the arrival time $t_{i}$ of the fluorescence light at the PMT pointing to the shower axis at an angle $\theta_{i}$ in the SDP is described by:

$$
t_{i}=\frac{R_{p}}{c} \tan \left(\frac{\chi_{i}-\chi_{0}}{2}\right)+T_{0}
$$

This relation can be fitted to data obtaining the free parameters $R_{p}, \chi_{0}$ and $T_{0}$. Together with the SDP these parameters fully define the event geometry.

Once the geometry is determined the conversation of measured photo-electrons (PE) into charged particles at shower

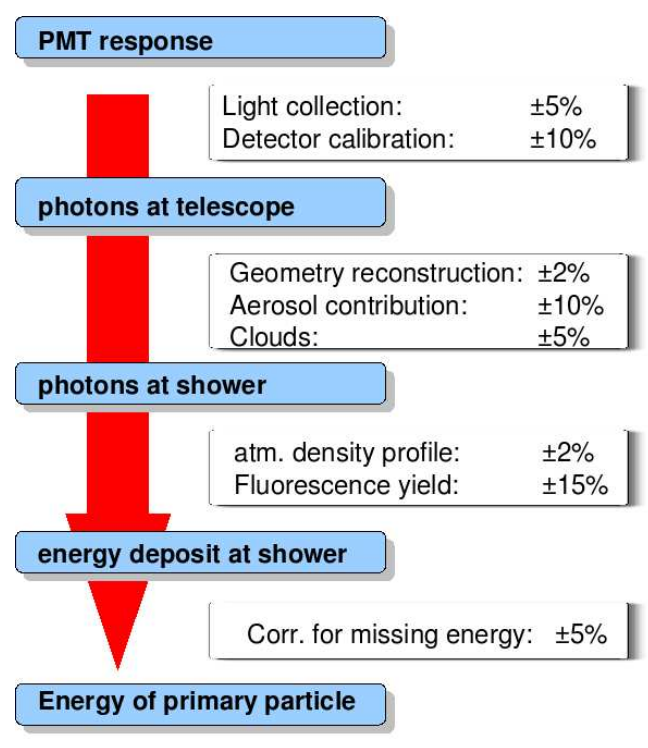

Fig. 4. Table of major sources for systematics in the FD event reconstruction. Adding the uncertainties in quadrature results in a $25 \%$ uncertainty in the reconstruction of the primary energy.

depth has to be done. This process includes light propagation properties of the atmosphere as well as understanding of the PMT response. The major systematic uncertainties stem from this conversion process and are listed in fig. 4. Firstly, the PE are translated into photons at the camera using the calibration constants. The light collection efficiency of the PMTs is known within 5\% uncertainty whereas the calibration constants can be measured with an uncertainty of $10 \%$. The backpropagation of the photons from the camera to the shower axis depends on the shower geometry. The influence of the geometry reconstruction accuracy on the conversion is less than $2 \%$. A major role is played by the weather conditions. Clouds can absorb and scatter light. This makes it important to monitor the cloud coverage. The systematic influence on the final energy is about $5 \%$. The absorption of aerosols can be determined to be $10 \%$.

The number of emitted photons at shower axis has to be converted to the energy deposit of charged particles at a certain shower depth. Hereby it is important to know the exact atmospheric density profile and the fluorescence yield. The density can be assumed to be known by $2 \%$, whereas the major impact on the systematics of $15 \%$ comes from the fluorescence yield. At this stage one is able to plot the energy loss $d E / d X$ versus slant atmospheric depth of the detected shower. This so called shower profile can be fitted by a Gaisser-Hillas parameterization. The depth of the shower maximum $X_{\max }$ is an indicator of the primary mass, whereas the integral of the profile is a measure for the total electromagnetic energy of the shower. This measure can be considered as lower bound for the energy of the primary particle. The amount of invisible energy which goes into muons and neutrinos depends on the shower development and is subject of fluctuations. For protons this correction is about $5 \%$, for iron induced showers the mean correction is about $15 \%$. Uncertainties from missing energy 


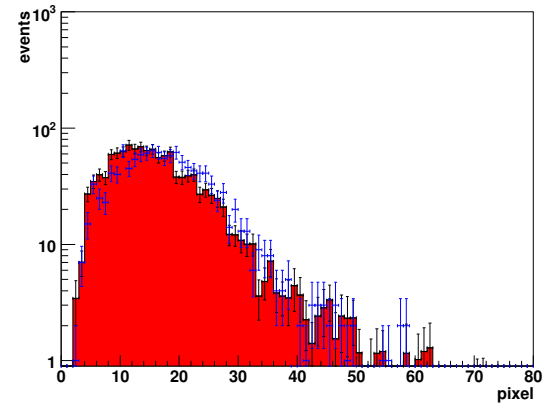

(a) Number of triggered pixels per event

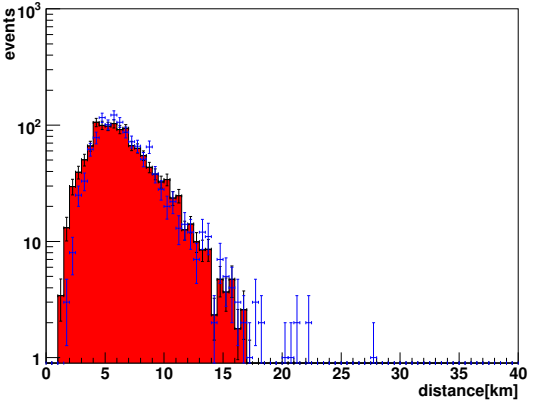

(b) Distance of shower axis to telescope

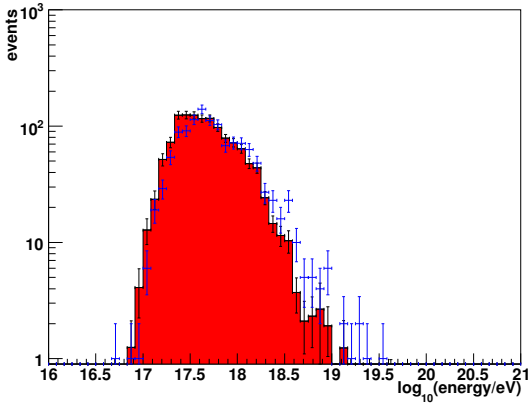

(c) reconstructed energy

Fig. 5. Data-MC comparison, after standard quality cuts. In red is shown the MC prediction, the blue crosses label the observed data

corrections can be assumed to be smaller than $10 \%$.

\section{RECONSTRUCTION PERFORMANCE}

In general, the data analysis relies on the accuracy of the reconstruction algorithm. To validate the reconstruction algorithm itself a simulation-reconstruction chain was created. A set of mono-energetic CORSIKA showers has been generated in steps of 0.5 in $\log _{10}$ (energy). For this dedicated study the core locations of the showers have been placed along a $2^{\circ}$ slice in the middle of the field of view of bay 4 in Los Leones. This allows us to investigate in detail the dependency of the reconstruction performance as function of energy and distance from the telescope. The simulation-reconstruction chain was applied including full atmospheric propagation, detector and trigger simulation. The reconstructed and generated properties have been compared. Therefore the RMS of the pull

\section{rec. observable -true observable}

true observable

was analyzed as function of the energy and distance of the EAS to the telescope. More details especially on the high energy performance can be found in [1]. For sake of completeness the results of the dedicated MC-simulationreconstruction study are listed in table I, a detailed description of the study can be found, i.e. in [8]

\begin{tabular}{l|c|c} 
& \multicolumn{2}{|c}{ shower core was } \\
RMS of & near & far away \\
\hline Zenith & $1^{\circ}$ & $2^{\circ}$ \\
Azimuth & $1^{\circ}$ & $4^{\circ}$ \\
$\mathrm{R}_{\text {core-eye }}$ & $100 \mathrm{~m}$ & $900 \mathrm{~m}$ \\
$X_{\text {max }}$ & $30 \mathrm{~g} / \mathrm{cm}^{2}$ & $50 \mathrm{~g} / \mathrm{cm}^{2}$ \\
Energy & $6 \%$ & $10 \%$ \\
\hline
\end{tabular}

TABLE I

Monocular reconstruction performance, according to [8]

\section{DATA-MC COMPARISON}

An independent way to validate the MC simulation itself is to generate the events according to a realistic flux and compare the observable distribution at reconstruction level. The aim of this comparison is not to determine the exact shape of the physical flux, but to prove that the simulation represents the reality. Thus, an $E^{-3}$ flux is a good assumption and the absolute normalization was fitted to the measured flux from the HiRes experiment [7]. So far, we have not included the influence of a possible flux suppression to the observable distribution due to the GZK cut-off. To cover the whole parameter range in an efficient way the energies of the simulated events have been generated according to an $E^{-2}$ power-law and the core positions have been placed only on those areas with a non-negligible efficiency. The physical flux was established using the method of importance sampling ([5], [6]), including an event-wise re-weighting for flux, energy, energy dependent area and lifetime. The data set chosen was FD-data from the period 2004-2005. The presented comparison focuses on events triggered in telescope 4 Los Leones. Standard quality cuts have been applied. The lifetime has been estimated from the noise-rate, as demonstrated in fig. 6.

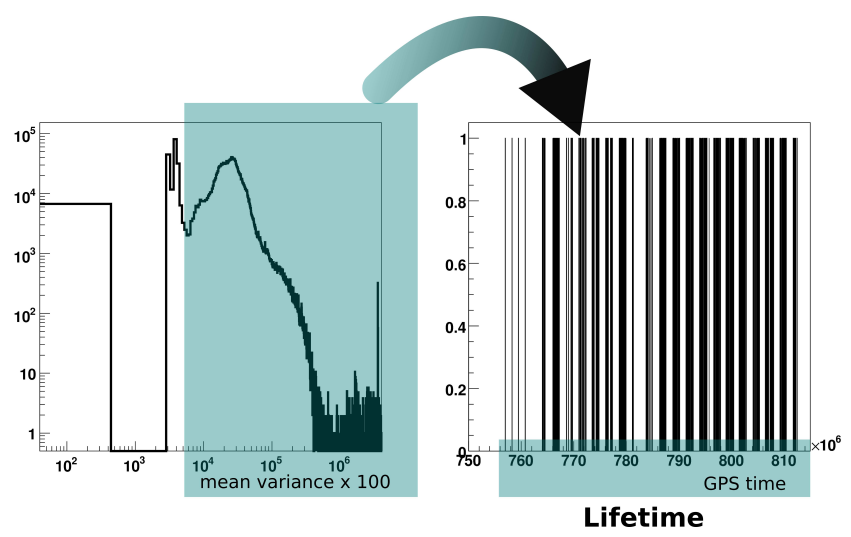

Fig. 6. Using background noise to estimate the lifetime of the detector 

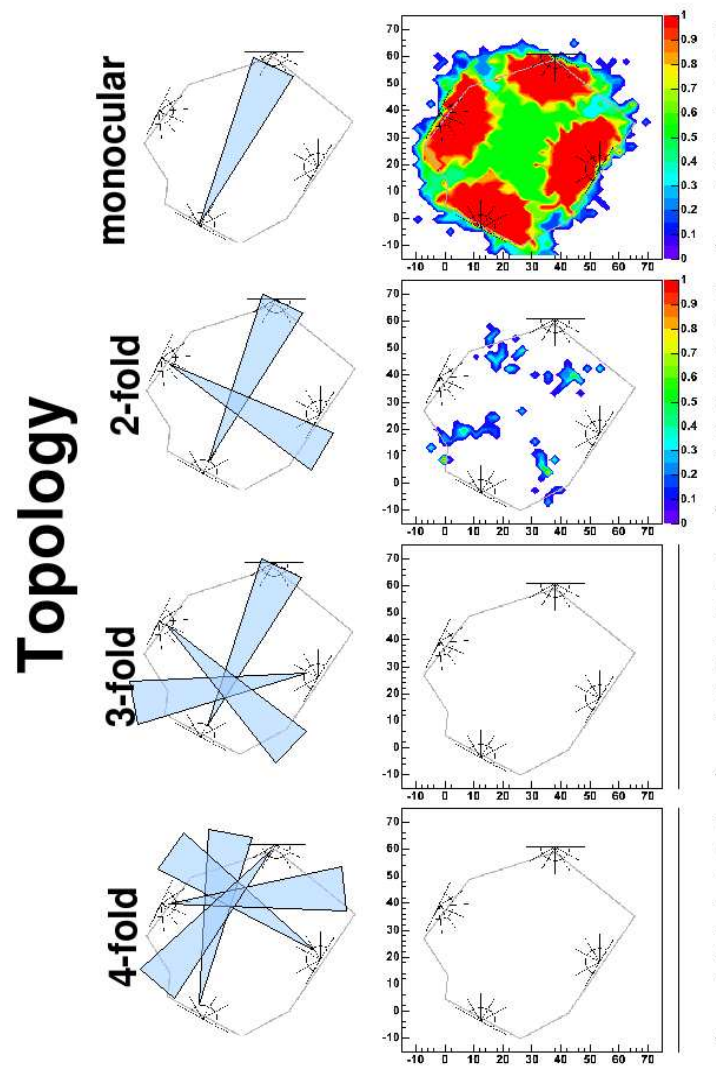

$10^{18} \mathrm{eV}-10^{18.5} \mathrm{eV}$
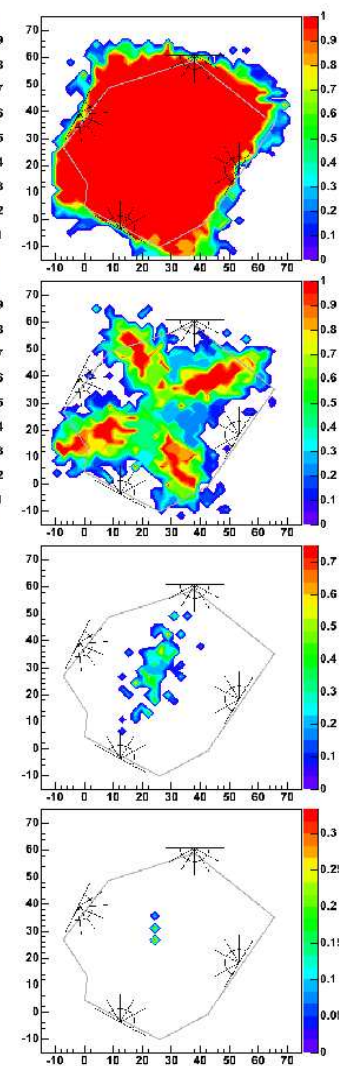

$10^{18.5} \mathrm{eV}-10^{19} \mathrm{eV}$
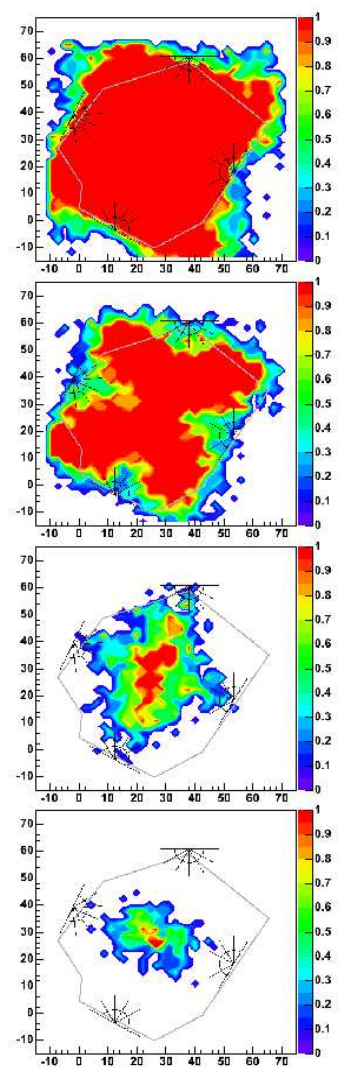

$10^{19} \mathrm{eV}-10^{19.5} \mathrm{eV}$
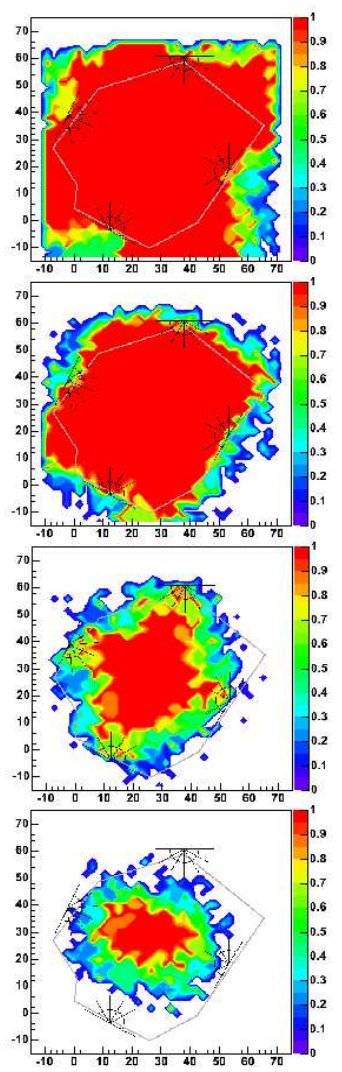

$10^{19.5} \mathrm{eV}-10^{20} \mathrm{eV}$

Fig. 7. Trigger efficiency for various event topologies as function of the core location on ground for increasing energies.

Every 30 seconds the background noise of each telescope is read out. In case the high voltage (HV) is off there is no noise visible. If the $\mathrm{HV}$ is ramped up the noise-level due to the night sky with open shutters differs significantly from the situation with closed shutters. Time integration of all periods with high noise-rate gives a possibility to estimate the lifetime for a specific telescope from data. As examples of the dataMC comparisons, fig. 5(a) shows the distributions of triggered pixels per event, fig 5(b) the distance of the shower-axis to the telescope and fig. 5(c) the reconstructed energy distribution. The MC prediction is shown as red histogram, the black error bars are the statistical uncertainties. The blue crosses represent the data distribution. Obviously, all MC and data observable distributions agree quite good in shape. It is interesting to note that the absolute normalization agrees within 10-20\%.

\section{TOPOLOGIES}

Besides FD monocular detection it is possible that a shower is seen and can be reconstructed by two (2-fold event) or 3 telescopes (3-fold event). When Loma Amarilla gets into operation by 2007 also 4 -fold events are expected. The possibility to see a shower by more than one telescope is of course dependent on the brightness (energy) of the shower and the geometry. Here we study the trigger efficiencies of multi-fold events. For this the MC-set was used and the core position was chosen in a square around the Auger array.
Fig. 7 shows the efficiency to trigger a 1-,2-,3- or 4-fold event at different core positions. The efficiency is studied at increasing energies. The calculated aperture can be folded with the individual lifetime of each telescope to obtain the corresponding exposure. Therefore, the method of importance sampling was applied again to represent a physical flux. The contour plot in fig. 8(a) shows the expected exposure for the data period 2004-2005 for stereo events. This exposure is dominated by the fact that Los Morados came in operation in mid 2005, thus most stereo events are expected to be seen by Los Leones and Coihueco. An example of a stereo event is shown in fig. 8(b). This event was also seen by the SD array. The reconstructed energies and geometries for stand alone reconstruction agree to each other. The contour plot in fig. 9(a) shows the expected exposure for the data period 20042005 for 3-fold events. An example of a 3-fold event is shown in fig. 9(b). Here the reconstructed energy and zenith angle is printed using just monocular reconstruction.

\section{CONCLUSION}

The Pierre Auger Observatory is still under construction. First data has been analyzed and presented. The fluorescence detectors play an important role, as they allow to calibrate the energy scale of the surface detector array in a modelindependent way. Moreover the FD-data was used in this analysis to validate the simulation-reconstruction chain. The 


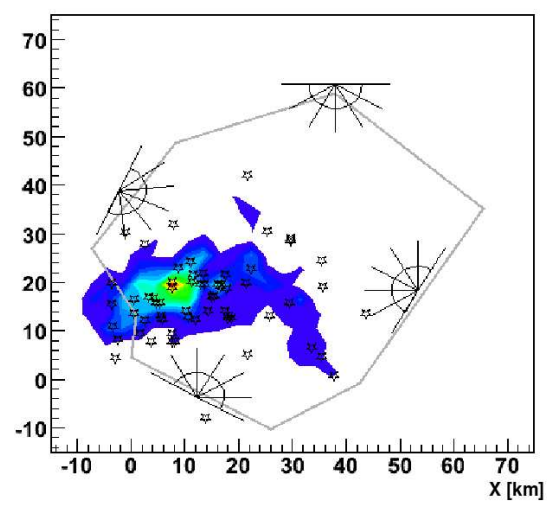

(a) Exposure for stereo events, compared to data

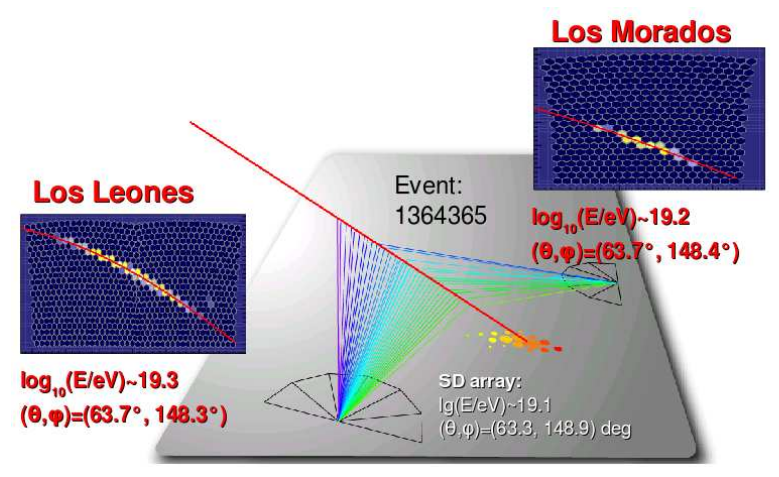

(b) A 2-fold event

Fig. 8. 2-fold exposure and data

overall systematic uncertainty in the fluorescence energy scale is estimated to be $25 \%$. A dedicated MC study of the reconstruction chain shows that the uncertainties of the energy reconstruction algorithm is about $10 \%$. To show that the simulation represents the data taken by the experiment, a realistic flux was simulated using the method of importance sampling. Various simulated observable distributions have been compared to the data and show good agreement in shape and normalization. The simulated data can also also used to calculate the aperture for multifold topologies. Folding with the lifetime bay-wise and applying importance sampling the exposure footprint is in good agreement with the 2-fold and 3fold event distributions detected. Operation of Loma Amarilla in 2007 opens up the promising opportunity to detect the 4fold events. A first data set of Auger has been analyses and the physics shows promising results ([3]). It was the intention of this work to present the potential of the Auger-FD and show the reliability of the FD-simulation and -reconstruction to understand the detector performance.

\section{ACKNOWLEDGMENT}

The work was supported by the German Ministry for Education and Research (BMBF). The author would like to thank the colleagues the Auger Collaboration for maintenance

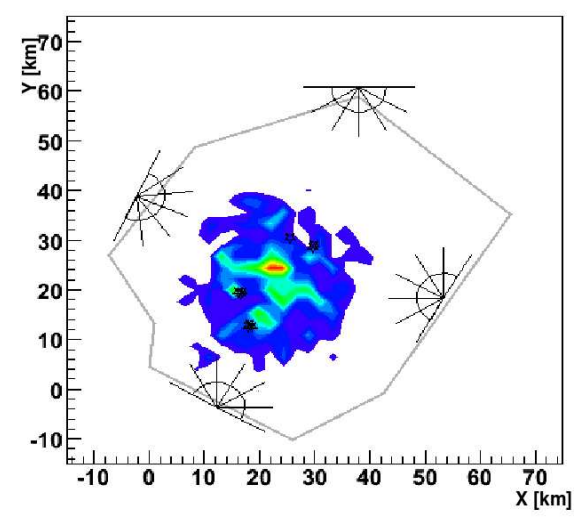

(a) 3-fold exposure compare to data

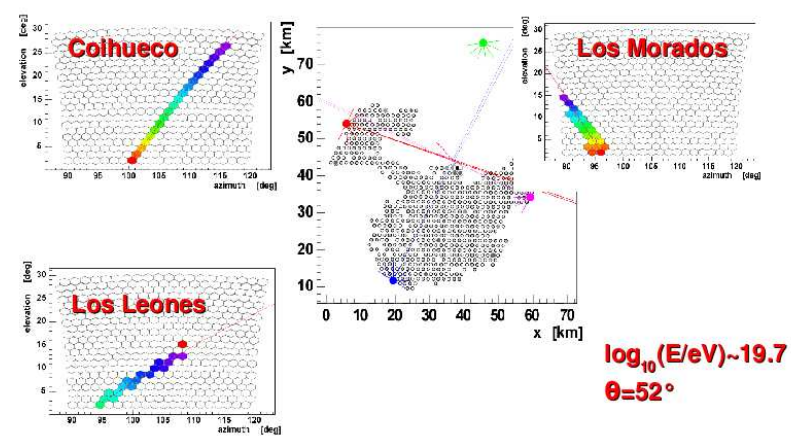

(b) A measured 3-fold event

Fig. 9. 3-fold exposure and data

and discussion, especially the author would like to thank the Auger group in Wuppertal. This work has profited a lot of K.-H. Kampert, N. Nierstenhöfer, L. Perrone, J. Rautenberg, M.Risse, S. Robbins and V. Scherini.

\section{REFERENCES}

[1] V.Scherini, Performance of the Pierre Auger Detector at the highest energies, Proceedings ECRS 2006, Lisbon

[2] F.Salamida, Hybrid Detection Aperture of the Pierre Auger Observatory, Proceedings ECRS 2006, Lisbon

[3] P.Mantsch, The Pierre Auger Observatory progress and first results, Proceedings ICRC 2005, Pune, vol 10, p.115

[4] H.Geenen et al., Validation of the real and simulated data of the Pierre Auger fluorescence telescopes, Proceedings ICRC 2005, Pune

[5] G.C. Hill, Application of Importance Sampling to the atmospheric Muon Generator, basiec, AMANDA-IR/20000904 (2000)

[6] G.C. Hill, An introduction to efficient simulation techniques, (1996), http://docushare.icecube.wisc.edu/docushare/dsweb/View/Collection2690

[7] Andreas Zech et al., A Measurement of the UHECR Spectrum with the HiRes FADC Detector, astro-ph/0409140 (2004)

[8] L. Perrone, Auger FD: Detector response to simulated showers and real event topologies, Proceedings CRIS 2004 